\title{
Simulation of Motion Locus in Bipolarity Double-nozzle Electrospinning
}

\author{
Haiying Du, ${ }^{1,2,3}$ Yanhui Sun, ${ }^{3,4 *}$ Jing Wang, ${ }^{3 * *}$ \\ Xiaogan $\mathrm{Li},{ }^{3}$ Qiang Shao, ${ }^{2}$ and Zhenghua $\mathrm{Liu}^{3}$ \\ ${ }^{1}$ Key Laboratory of Microelectronic Devices \& Integrated Technology, Institute of Microelectronics, \\ Chinese Academy of Sciences, Beijing 100029, China \\ ${ }^{2}$ College of Mechanical and Electronic Engineering, Dalian Minzu University, Dalian 116600, China \\ ${ }^{3}$ School of Electronic Science and Technology, Dalian University of Technology, Dalian 116023, China \\ ${ }^{4}$ College of Information \& Communication Engineering, Dalian Minzu University, Dalian 116600, China
}

(Received May 26, 2018; accepted October 30,2018)

Keywords: electrospinning, hetero-nanofibers, simulation, bipolarity double nozzle, Runge-Kutta algorithm

Electrospinning was used to synthsize nanofibers to be used as gas sensing materials. An electrospinning device with double nozzles of opposite polarities was designed for the synthesis of $\mathrm{SnO}_{2} / \mathrm{In}_{2} \mathrm{O}_{3}$ hetero-nanofibers in our experiments. It also included two syringes with different solutions connected to the bipolarity nozzles, and a collection plate connected to the ground. In this work, the Matrix Laboratory (MATLAB) software was used to simulate the motion locus of the bipolarity double-nozzle electrospinning process. The charged solutions were simulated as multiply charged and discretized particles. Then, force analysis was carried out for the charged particles composing the electrospun fibers, and a molecular dynamics model was built. The motion locus of the opposite electrically charged particles was calculated and simulated on the basis of the Runge-Kutta algorithm. Nanofibers with opposite polarities were attracted to and intertwined with each other and then descended to the collection plate because of gravity. The motion locus of the organic composite nanofibers synthesized using the bipolarity double-nozzle electrospinning device is shown to be identical to the simulated motion locus.

\section{Introduction}

The electrospinning technique is a simple and easy method of fabricating ultrathin nanofibers. It was mainly applied to fabricating pure organic polymers in previous years. ${ }^{(1,2)}$ Recently, this technique has been extended to the synthesis of inorganic nanofibers, inorganic composite nanofibers, and organic/inorganic composite nanofibers, such as $\mathrm{SnO}_{2},{ }^{(3)}$ $\mathrm{In}_{2} \mathrm{O}_{3},{ }^{(4)} \mathrm{WO}_{3},{ }^{(5)} \mathrm{ZnO},{ }^{(6)} \mathrm{Co}_{3} \mathrm{O}_{4},{ }^{(7)} \mathrm{BaTiO}_{3},{ }^{(8,9)} \mathrm{CuO} / \mathrm{SnO}_{2},{ }^{(10)} \mathrm{SnO}_{2} / \mathrm{In}_{2} \mathrm{O}_{3},{ }^{(1)} \mathrm{TiO}_{2} / \mathrm{Al}_{2} \mathrm{O}_{3},{ }^{(12)}$ $\mathrm{In}_{2} \mathrm{O}_{3} /$ carbon fibers, ${ }^{(13)}$ and polyaniline/carbon black. ${ }^{(14,15)}$ Furthermore, electrospinning has proven to be a highly effective technique for controlling the synthesis of one-dimensioned (1D)-

\footnotetext{
*Corresponding author: e-mail: syh@dlnu.edu.cn

** Corresponding author: e-mail: wangjing@dlut.edu.cn https://doi.org/10.18494/SAM.2018.2008
} 
morphology nanostructures including nanofibers, nanotubes, nanowires, ${ }^{(16)}$ nanoribbons, ${ }^{(17)}$ and other novel structures. ${ }^{(18-21)}$

A typical electrospinning system includes a high-voltage power supply, a syringe with a nozzle connected to the positive electrode and a collector connected to the negative electrode. In the process of electrospinning, a prepared organic solution (or a mixed solution with an inorganic material) is put into the syringe. An electrical field is created between the nozzle and the collector when a high voltage is applied. The solution droplet at the tip of the nozzle will form a Taylor cone through the action of multiple forces, such as electric field force, Coulomb force, gravity, surface tension, and viscosity force of the solution. ${ }^{(22-24)}$ When the supplied voltage reaches a critical level at which the electric field force can overcome the solution surface tension, the solution droplet will be ejected. A jet stream of the solution moves to the electrically grounded collection plate and forms organic nanofibers. Inorganic nanofibers can be obtained by annealing. With the maturation of electrospinning technology, many solutions have been developed in order to synthesize fancy spinning fibers with enhanced performances. Unfortunately, the electrospinning process is extremely complex. Many factors affect the motion locus and morphology of nanofibers in the electrospinning process, including solution properties (such as viscosity, concentration, surface tension, and electrical conductivity) and operating parameters (such as applied voltage, electric field distribution, magnetic field distribution, flow rate of the solution, distance between the nozzle and the collector, temperature, humidity, pressure, and other environmental factors). ${ }^{(25-28)}$ Some researchers concentrate on studying the motion locus simulation and emulation of nanofibers for the optimization of the electrospinning parameters and control of the electrospinning process, which will enable us to control the morphology and structure of electrospinning nanofibers effectively. ${ }^{(23,29-32)}$ The simple and effective numerical simulation and modelling not only represent an alternative solution to obtaining the same result with a reduced number of experimental tests but also enable the optimization of simulation parameters to guide the electrospinning process. ${ }^{(33)}$

A modified electrospinning system including double nozzles with opposite polarities was designed and different material nanofibers were synthesized simultaneously in our laboratory. ${ }^{(34,35)}$ Figure 1 shows a sketch of a bipolarity double-nozzle electrospinning device. We can see that

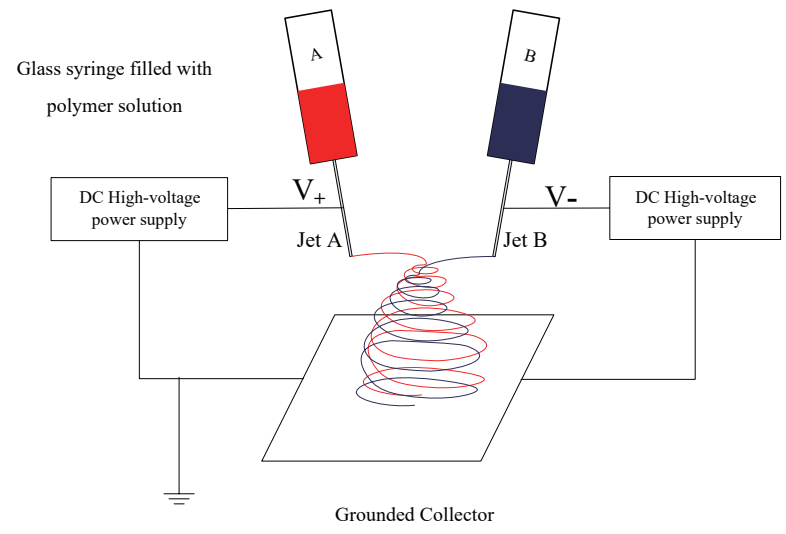

Fig. 1. (Color online) Sketch of bipolarity double-nozzle electrospinning device. 
two syringes with nozzles containing different solutions were put in opposite electrical fields. The two nozzles were connected to a positive polarity and a negative polarity, and the collection plate had zero potential. In this work, the forces of the charged particles ejected from the two nozzles of opposite polarities were analyzed and calculated, and a molecular dynamics model was built. The motion locus of the charged particle was calculated and simulated on the basis of the Runge-Kutta algorithm using the Matrix Laboratory (MATLAB) software. ${ }^{(36)}$ Unlike single-nozzle electrospinning, the charged and discretized particles from two nozzles of opposite polarities were subjected to an attractive force from opposite-polarity particles. Under the combination of the electrical field force, Lorentz force, gravity, and surface tension of the solution, two types of nanofiber ejected from their respective nozzles moved in opposite directions owing to a strong electric field force and interweaved together, finally resulting in a net of composite hetero-nanofibers on the collection plate. The optimum experimental parameters of bipolarity double-nozzle electrospinning are obtained by simulation. Simulation provides a more effective solution for synthesizing composite hetero-nanofibers.

\section{Modeling}

In the electrospinning process with double nozzles in a bipolarity system, the positively charged solution is affected by the electrical field force of the negative electrode and zero potential, and the jet stream ejected from the positively charged solution moves toward the negative electrode. The ejected jet stream is simulated as comprising many discretized particles and the charged particles are treated as a series of infinitesimal and closely adjacent mass points ignoring its mass. ${ }^{(37)}$ Figure 2 shows a model of a jet stream composed of particles in a bipolarity double-nozzle electrospinning system. During electrospinning, the charged particle motion is divided into two stages: the linear motion stage and the unstable motion stage. ${ }^{(31)}$ Under high-voltage electrostatic fields from the two directions, vertical and horizontal, a

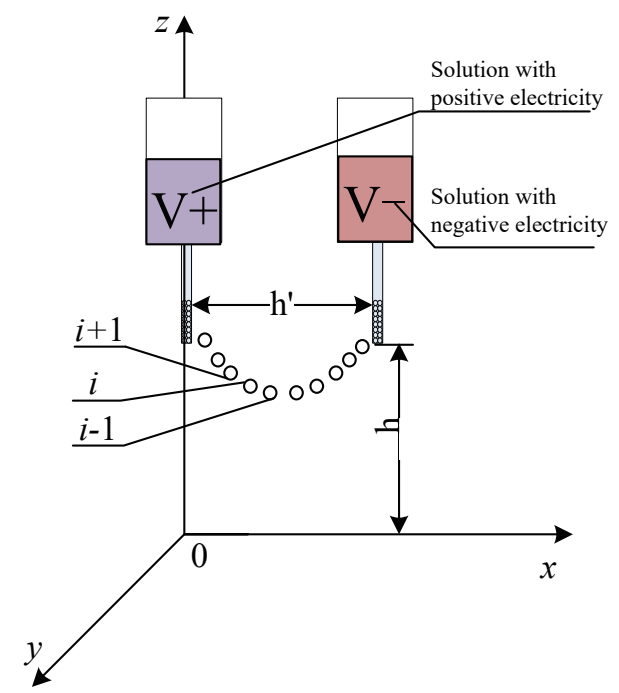

Fig. 2. (Color online) Model of jet stream composed of particles in the bipolarity double-nozzle electrospinning system. 
jet stream is ejected from the nozzle and accelerates along a straight line. ${ }^{(38)}$ During the acceleration motion, the viscous resistance of solution particles becomes high. When the viscous resistance reaches or exceeds the electrical field force, the jet stream will become unstable. At the same time, the acceleration of the stream becomes very small, and a weak perturbation will make the stream diverge from its equilibrium position; then the unsteady motion stage begins. The motion locus of nanofibers in electrospinning is mainly determined by the force states of the particles in the above two stages. ${ }^{(39)}$

\subsection{Discretization of jet stream}

The jet stream ejected from the positive nozzle is simulated as comprising many discrete positively charged particles. These charged particles are held together with a Maxwell gooey substance, where the discrete charged particles are considered as viscoelastic elements connected unstably in accordance with Earnshaw's theorem. ${ }^{(40)}$ Denote a particle in the continuous jet stream as $i$, and the particles before and after it as $i+1$ and $i-1$, respectively. The subscripts $p$ and $q$ are used to express the variable parameters related to $i+1$ and $i-1$ particles, respectively. The coordinate of particle $i\left(x_{i}, y_{i}, z_{i}\right)$ is a function of time $t$. Denote the length of the chain (the distance between any two adjacent particles in the space) between $i$ and $i+1$ as $l_{p i}$, and between $i$ and $i-1$ as $l_{q i}$.

$$
\begin{aligned}
& l_{p i}=\left[\left(x_{i+1}-x_{i}\right)^{2}+\left(y_{i+1}-y_{i}\right)^{2}+\left(z_{i+1}-z_{i}\right)^{2}\right]^{1 / 2} \\
& l_{q i}=\left[\left(x_{i}-x_{i-1}\right)^{2}+\left(y_{i}-y_{i-1}\right)^{2}+\left(z_{i}-z_{i-1}\right)^{2}\right]^{1 / 2}
\end{aligned}
$$

The distance between any two particles with the same polarity $i$ and $j\left(R_{i j}\right)$ can be determined using Eq. (3). The distance between any two particles of opposite polarities $i$ and $z, z \in(0 \sim N)\left(R_{i z}\right)$ can be determined using Eq. (4).

$$
\begin{aligned}
& R_{i j}=\left[\left(x_{i}-x_{j}\right)^{2}+\left(y_{i}-y_{j}\right)^{2}+\left(z_{i}-z_{j}\right)^{2}\right]^{1 / 2} \\
& R_{i z}=\left[\left(x_{z}-x_{i}\right)^{2}+\left(y_{z}-y_{i}\right)^{2}+\left(z_{z}-z_{i}\right)^{2}\right]^{1 / 2}
\end{aligned}
$$

\subsection{Force analysis}

The force of one particle in a jet stream ejected from the positive-polarity nozzle was first analyzed. Figure 3 shows the force analysis of a positive particle. Under the vertical and horizontal electrostatic fields, the charged particle will be subjected to multiple forces including electrical field force $\left(f_{e}\right)$ between two opposite-polarity nozzles, Coulomb force $\left(f_{c}\right)$ between two particles, solution viscosity force $\left(f_{v}\right)$, solution surface tension $\left(f_{s}\right)$, gravity $\left(f_{z}\right)$, and Lorentz 


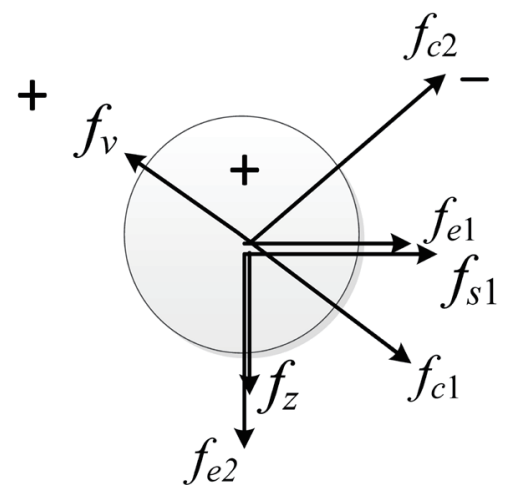

Fig. 3. (Color online) Force analysis of a positively charged particle.

force $\left(f_{l}\right)$ caused by the magnetic field. During movement, the combined force on the charged particle will change with time and location, and the motion locus is unstable. When a positively charged nanofiber encounters a negatively charged nanofiber at a given space, the opposite charges neutralize each other and neutral nanofibers are formed. The neutral nanofibers fall to the collection plate owing to gravity and form a network composed of two kinds of nanofibers.

The gravity force of the positively charged particle $\left(f_{z}\right)$ is expressed as

$$
f_{z}=m g \vec{j}
$$

where $m$ denotes the mass of the particle and $g(9.8 \mathrm{~N} / \mathrm{mg})$ is the acceleration due to gravity.

The electrical field force of the positively charged particle is composed of two parts. One is the electrical field force between the positive electrode and the zero potential collector $\left(f_{e l}\right)$, as determined using Eq. (6), where $V_{0}$ is the electrostatic pressure of a high-voltage electrical field.

$$
f_{e 1}=-e \frac{V_{0}}{h} \vec{k}
$$

The other one, $f_{e 2}$, is the electrical field force between the positive and negative nozzles. In this electrical field, the charged particle at the edge of the positive-polarity and negativepolarity electrical field will move along the tangent direction of the field. Thus, the force of the charged particle is complicated. In the vertical direction, the effect of the positive and negative electrodes can be neutralized, so the force is weak and can be ignored. In the horizontal direction, the magnitude of the electrical field force increases as distance decreases. The electrical field force disappears when the positively and negatively charged particles collide. This part of the electrical field force is expressed as

$$
f_{e 2}=2 e \frac{V_{0}}{h_{1}\left(h-y_{i}\right)} \vec{i},\left(x<\frac{h_{1}}{2}\right),
$$


where $h$ and $h_{1}$ are the distances between one nozzle and the collector, and between the two nozzles, respectively, and $y_{i}$ is the coordinate of the charged particle at a given moment. Figure 4 shows a schematic of the horizontal-direction electric field force of the charged particle in the bipolarity system.

The Coulomb force $f_{c 1}$ of other particles with the same electrical polarity acting on particle $i$ is calculated as

$$
f_{c 1}=\sum_{\substack{j=1 \\ j \neq i}}^{N} \frac{k e^{2}}{R_{i j}^{2}}\left[\frac{x_{i}-x_{j}}{R_{i j}} \vec{i}+\frac{y_{i}-y_{j}}{R_{i j}} \vec{j}+\frac{z_{i}-z_{j}}{R_{i j}} \vec{k}\right],
$$

where $k$ is the coefficient of the Coulomb force and $\vec{i}, \vec{j}$, and $\vec{k}$ are the unit vectors in three directions. The Coulomb force $f_{c 2}$ of other particles with the opposite electrical polarity acting on particle $i$ is calculated as

$$
f_{c 2}=\sum_{z=1}^{N} \frac{k e^{2}}{R_{i z}^{2}}\left[\frac{x_{i}-x_{z}}{R_{i z}} \vec{i}+\frac{y_{i}-y_{z}}{R_{i z}} \vec{j}+\frac{z_{i}-z_{z}}{R_{i z}} \vec{k}\right] .
$$

The diameters of the nanofibers were considerably affected by the viscosity of the organic solution in the electrospinning process. The viscosity force acting on the particles is an important parameter affecting the electrospinning path. The electrospinning fibers seem to consist of many charged particles held together with the Maxwell gooey substance. ${ }^{(40,41)}$ The Maxwell gooey substance is elastic and viscous, just like a spring-damper model, and it should satisfy

$$
\frac{1}{E} \frac{d \sigma}{d t}+\frac{\sigma}{\eta}=\frac{d \varepsilon}{d t}
$$

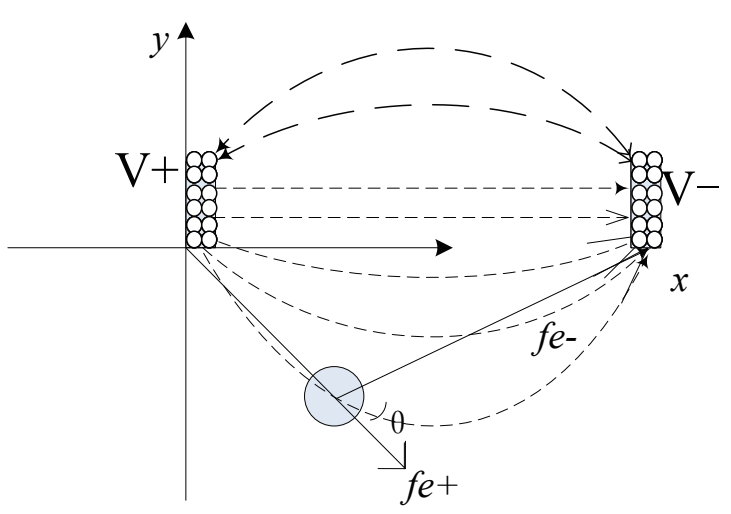

Fig. 4. Schematic of horizontal-direction electric field force of the charged particle in the bipolar system. 
$\eta$ is the viscosity coefficient of the damper, $E$ is the elastic coefficient of the spring, and $\sigma$ and $\varepsilon$ represent the pressure and stress of the spring-damper model, respectively.

We define $\sigma$ as the stress on particle $i$, and it should be applied between particle $i$ and its neighboring particles $i+1$ and $i-1$. Stress $\sigma$ should satisfy

$$
\begin{aligned}
& \frac{d \sigma}{d t}=G \frac{d l}{l d t}-\frac{G}{\mu_{v}} \sigma \\
& \frac{d \sigma_{p i}}{d t}=G \frac{1}{l_{p i}} \frac{d l_{p i}}{d t}-\frac{G}{\mu_{v}} \sigma_{p i}, \\
& \frac{d \sigma_{q i}}{d t}=G \frac{1}{l_{q i}} \frac{d l_{q i}}{d t}-\frac{G}{\mu_{v}} \sigma_{q i}
\end{aligned}
$$

where $t$ is time, and $G$ and $\mu_{\nu}$ are the elastic coefficient and viscosity coefficient, respectively.

Suppose the initial diameter of the jet stream is $a_{0}$. In accordance with the law of mass conservation, we have

$$
\pi a_{p i}^{2} l_{p i}=\pi a_{0}^{2} L=\pi a_{q i}^{2} l_{q i}
$$

$s f_{v}$ acting on particle $i$ can be calculated as

$$
\begin{aligned}
f_{v} & =\pi a_{p i}^{2} \sigma_{u i}\left[\frac{x_{i+1}-x_{i}}{l_{p i}} \vec{i}+\frac{y_{i+1}-y_{i}}{l_{p i}} \vec{j}+\frac{z_{i+1}-z_{i}}{l_{p i}} \vec{k}\right] \\
& -\pi a_{d i}^{2} \sigma_{d i}\left[\frac{x_{i}-x_{i-1}}{l_{q i}} \vec{i}+\frac{y_{i}-y_{i-1}}{l_{q i}} \vec{j}+\frac{z_{i}-z_{i-1}}{l_{q i}} \vec{k}\right] .
\end{aligned}
$$

The surface tension needed to keep the jet stream moving along a straight line can be determined by

$$
f_{S}=-\frac{a \pi k_{i}\left(r_{p i}+r_{q i}\right)^{2} / 4}{\left(x_{i}^{2}+y_{i}^{2}\right)^{\frac{1}{2}}}\left[\left|x_{i}\right| \operatorname{sign}\left(x_{i}\right) \vec{i}+\left|y_{i}\right| \operatorname{sign}\left(y_{i}\right) \vec{j}\right],
$$

where $a$ is the coefficient of surface tension, $k_{i}$ is the curvature of the jet stream at the location of particle $i$, and $k_{i}$ is calculated using the coordinates of $i+1, i, i-1$. 'sign $(x)$ ' is a symbolic function, the value of which is determined using

$$
\operatorname{sign}(x)=\left\{\begin{array}{l}
1(x>0) \\
-1(x<0) . \\
0(x=0)
\end{array}\right.
$$




\subsection{Simulation model}

According to Newton's Second Law, in the absence of an applied magnetic field and ignoring the effect of the external magnetic field (Lorentz force), the united force $f_{H}$ of the charged particle should satisfy

$$
f_{H}=f_{c 1}+f_{c 2}+f_{e 1}+f_{e 2}+f_{v}+f_{s}+f_{z}
$$

The motion locus of particle $i$ should satisfy Eq. (17) by simplifying Eq. (16).

$$
\begin{aligned}
m \frac{d^{2} \vec{r}_{i}}{d t^{2}} & =\sum_{\substack{j=1 \\
j \neq i}}^{N} \frac{k e^{2}}{R_{i j}^{3}}\left(\vec{r}_{i}-\vec{r}_{j}\right)+\sum_{z=1}^{N} \frac{k e^{2}}{R_{i z}^{3}}\left(\vec{r}_{i}-\vec{r}_{z}\right)-e \frac{V_{0}}{h} \vec{k}+2 e \frac{V_{0}}{h_{1}\left(h-y_{i}\right)} \vec{i} \\
& +\frac{\pi a_{p i}^{2} \sigma_{p i}}{l_{p i}}\left(\vec{r}_{i+1}-\vec{r}_{i}\right)-\frac{\pi a_{q i}^{2} \sigma_{q i}}{l_{q i}}\left(\vec{r}_{i}-\vec{r}_{i-1}\right) \\
& -\frac{a \pi\left(\vec{r}_{p i}+\vec{r}_{q i}\right)^{2}}{4 \sqrt{\left(x_{i}^{2}+y_{i}^{2}\right)}}\left[\left|x_{i}\right| \operatorname{sign}\left(x_{i}\right) \vec{i}+\left[\left|y_{i}\right| \operatorname{sign}\left(y_{i}\right) \vec{j}\right]+m g \vec{j}\right.
\end{aligned}
$$

Here, $\vec{r}_{i}$ is a coordinate vector of particle $i$ and $\vec{r}_{i}=x_{i} \vec{i}+y_{i} \vec{j}+z_{i} \vec{k}$. The wave amplitude of the particle $\left(\sqrt{x_{i}^{2}+y_{i}^{2}}\right)$ can be calculated using the coordinate $\left(x_{i}, y_{i}, z_{i}\right)$.

\section{Simulation of Electrospinning Process}

Using the MATLAB software, the molecular dynamics model of the charged particle motion locus was numerically calculated. On the basis of the Runge-Kutta algorithm, the simulation motion locus of the charged particles in the bipolarity nozzle electrospinning system can be drawn by selecting an appropriate step size and relative parameters.

The jet stream from the charged nozzle is taken to be many connected charged particles, the number of which is defined as $N$. The particle at the bottom of the jet stream is marked $i=1$ and the top particle is $i=N$. The particle number $N$ changes with the motion of the jet stream. At time $t$, if the distance between the $N$ th particle and the electrospinning nozzle is more than $h / 20000$, the particle number at the top of the jet stream is increased by 1 , that is $N$ $=N_{0}+1$. Meanwhile, an initial position is given to the added particle as $(0,0,6)$ (to simulate the heliciform motion locus of the jet stream, a disturbance is added to each particle). Then, the coordinate of a charged particle with time can be expressed as $\left[x_{N}=10^{-3} L \sin (\omega t), y_{N}=\right.$ $\left.10^{-3} L \cos (\omega t), z_{N}=49999 h / 50000\right]$, where $\omega$ is the disturbance frequency.

The parameters of a charged particle were determined by calculating its motion locus. Set $t=0$ as the initial time of the charged particles, the particle number of the jet stream as $N=2$, and the initial velocity of every particle as 0 . Set the surface charge density of particles as $\sigma_{d l}=0$ and $\sigma_{u N}=0$ and the length of the connected-particle chain as $l_{d l}=0$ and $l_{u N}=0$. 


\section{Analysis of Simulation and Experiment}

The electrical field orientation of the bipolarity double-nozzle electrospinning system is simulated. The collection plate is a square iron plate with a side length of $12 \mathrm{~cm}$ and a thickness of $2 \mathrm{~mm}$. The diameters and lengths of the two nozzles are both $0.5 \mathrm{~mm}$ and 1.5 $\mathrm{cm}$, respectively. The applied voltages on the two nozzles are $\pm 20 \mathrm{kV}$. The vertical distance between the nozzles and the collection plate is $h=5 \mathrm{~cm}$, and the distance between the two nozzles is $6 \mathrm{~cm}$. We can see from Fig. 5 that the nozzle on the left side of the space is subjected to $20 \mathrm{kV}$ and the nozzle on the right side is subjected to $-20 \mathrm{kV}$. The geometry model of the bipolarity double-nozzle electrospinning device and the electrical field orientation of the bipolarity double-nozzle electrospinning system are shown in Figs. 5(a) and 5(b), respectively. A parallel electrical field with the direction from left to right is formed between two needles owing to the symmetry of the two nozzles with opposite polarities. From the results of the simulated analysis of the distribution of the electrical field, it can be inferred that the jet stream with a positive charge should be ejected from the left nozzle and shift to the right side with the negative charge under the action of the electrical field force, whereas the jet stream with the negative charge should shift to the left side.

Figures 6(a)-6(f) show the simulation motion loci of the jet stream produced using a bipolarity double-nozzle electrospinning device with the applied voltages of 12,20 , and $28 \mathrm{kV}$, respectively. The parameters used here are as follows: viscosity coefficient $\mu=10^{4} \mathrm{~kg} / \mathrm{ms}$, elastic modulus $G=10^{5} \mathrm{~kg} / \mathrm{ms}^{2}$, disturbance frequency $\omega=10^{-4} \mathrm{~s}^{-1}$, and coefficient of surface tension $\alpha=0.12 \mathrm{~N} / \mathrm{m}$. the vertical distance between the nozzles and the collector is $h=5 \mathrm{~cm}$, and the distance between two nozzles is $h_{1}=6 \mathrm{~cm}$.

Figures 6(a), 6(c), and 6(e) are the main plots of the simulation motion loci under the different applied voltages, obtained using a bipolarity double-nozzle electrospinning system. We find that the motion loci spiral downward to the collector in the early spinning process owing to the electrical field force between the nozzle and the collector being much greater than the coulomb force between the opposite charges in this stage. With increasing distance between the electrospinning fiber and the needle, the electrical field force decreases rapidly. The coulomb force between the opposite charges is greater than the electrical field force between the nozzle

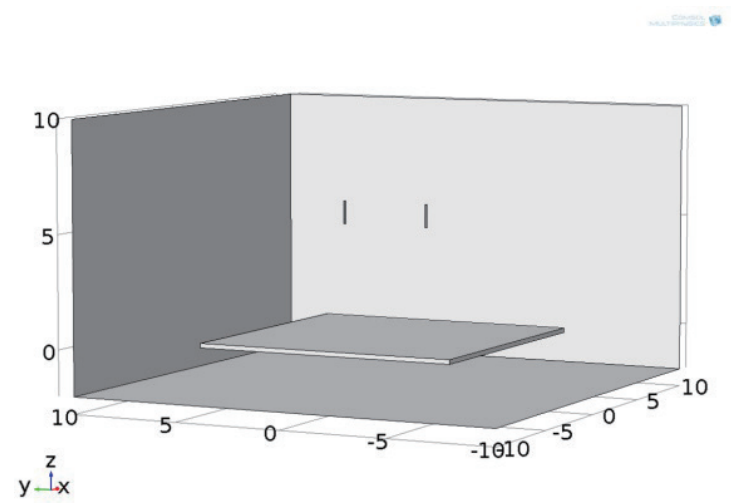

(a)

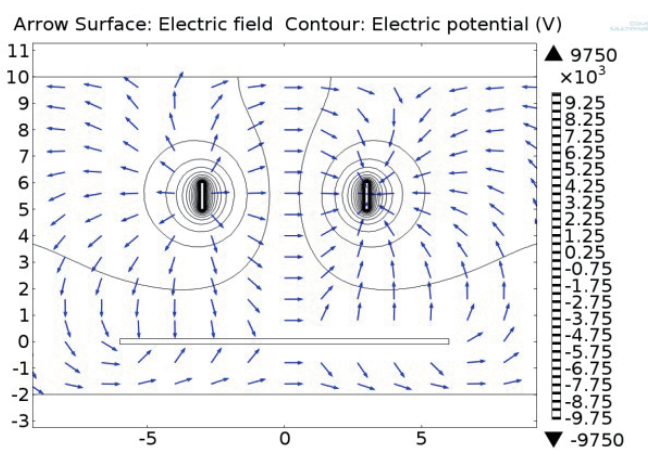

(b)

Fig. 5. (Color online) (a) Geometrical model. (b) Electrical field orientation of double-nozzle electrospinning device. 


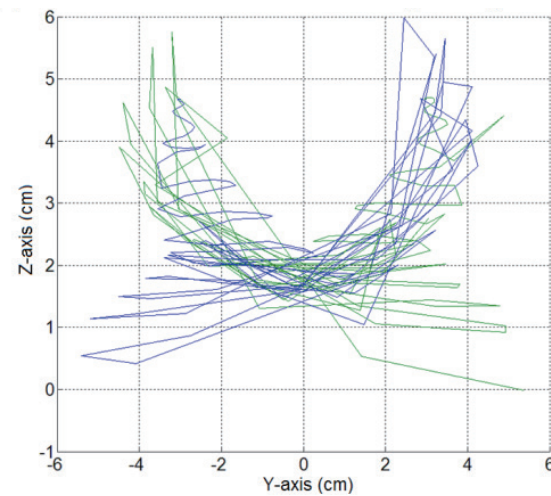

(a)

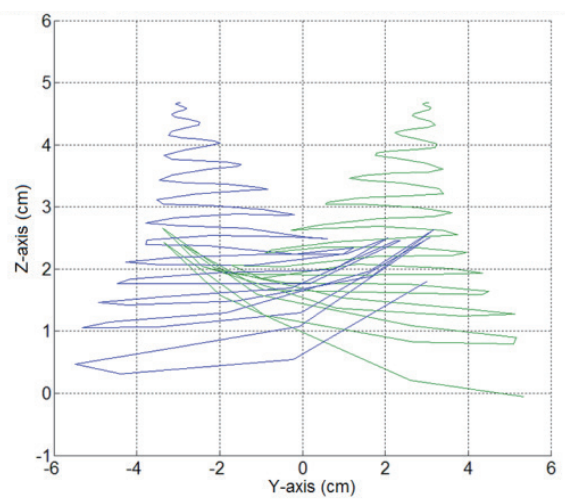

(c)

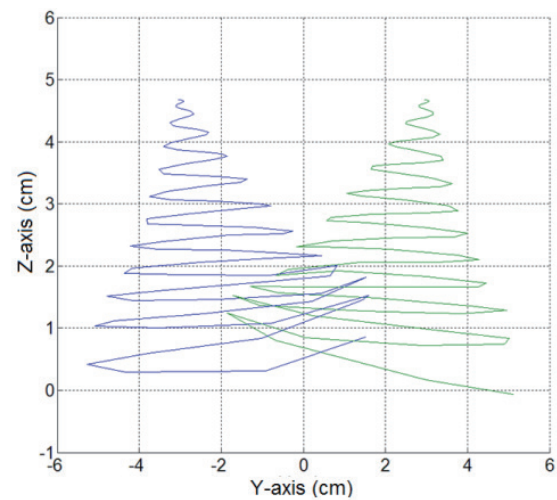

(e)

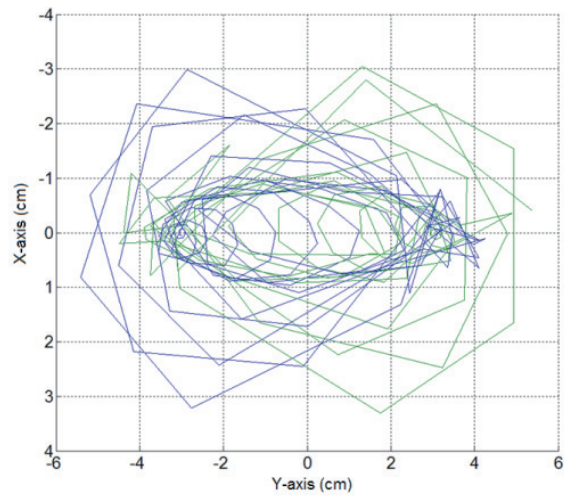

(b)

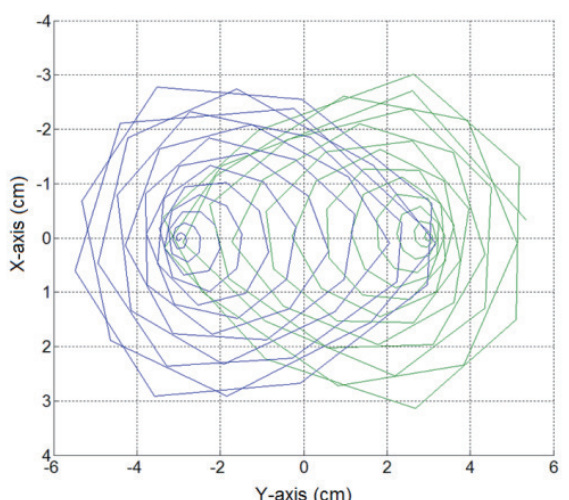

(d)

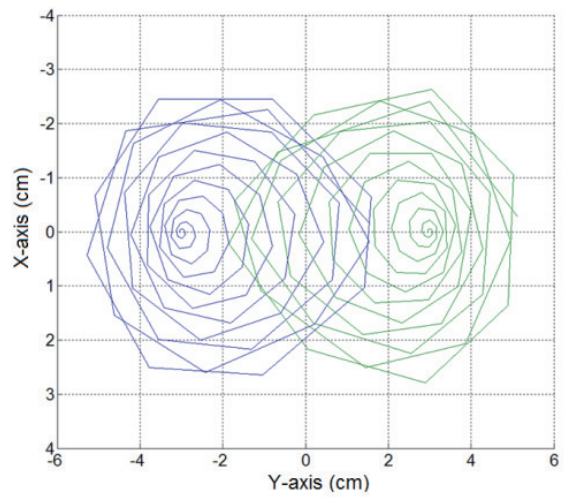

(f)

Fig. 6. (Color online) Plots of the simulated motion loci of the jet stream at different applied voltages produced using the bipolarity double-nozzle electrospinning system. (a) Main view at applied voltage of $12 \mathrm{kV}$. (b) Top view at applied voltage of $12 \mathrm{kV}$. (c) Main view at applied voltage of $20 \mathrm{kV}$. (d) Top view at applied voltage of $20 \mathrm{kV}$. (e) Main view at applied voltage of $28 \mathrm{kV}$. (f) Top view at applied voltage of $28 \mathrm{kV}$.

and the collector. The electrospinning fibers with opposite polarities move towards each other. When the electrospinning fiber ejected from the positive-polarity nozzle meets one from the negative-polarity nozzle, their opposite polarities attract and the fibers intertwine with each other. Then, the opposite charges neutralize each other and the intertwined fibers become 
neutral. The neutral nanofibers descend to the collector plate because of gravity. Comparing the simulation motion loci at the different applied voltages of 12,20 , and $28 \mathrm{kV}$, we find that the areas of interwoven electrospinning fibers of opposite polarities gradually decrease with increasing applied voltage. The distance among interwoven electrospinning fibers increases gradually. Consequently, $20 \mathrm{kV}$ is selected as the optimal applied voltage for synthesizing composite hetero-nanofibers using a bipolarity double-nozzle electrospinning system. With the same experimental parameters as the simulation parameters, it can been seen from Figs. $7(\mathrm{a})-7(\mathrm{c})$ that the electrospinning hetero-nanofiber trajectory presents similar motion loci to those of the simulation.

The electrospinning distance between nozzles is the important factor in the simulation of motion loci. The motion loci of fibers at electrospinning distances of 4, 5, and $6 \mathrm{~cm}$ between the two nozzles have been simulated at the optimal applied voltage of $20 \mathrm{kV}$. When the distance between the two nozzles is $4 \mathrm{~cm}$, we can see, from Fig. 8(a), that the simulated motion loci of fibers with opposite polarities are unstable and fibers attraction and interweaving occur quickly because of the rapidly increasing coulomb force. When the distance between the two nozzles increases to $5 \mathrm{~cm}$, the simulation motion loci of fibers with opposite polarities show attraction and interweaving within the distance of $4 \mathrm{~cm}$ from the nozzles. As a result, the two kinds of spinning fibers are sufficiently mixed to interweave together uniformly. Figures 8 (a)-8(f) show the simulated motion loci of the jet stream at different electrospinning distances between the two nozzles of the bipolarity double-nozzle electrospinning system. Figures 9(a)-9(c) show photographs of electrospinning hetero-nanofiber trajectories for different distances between the two nozzles of the bipolarity double-nozzle electrospinning system. We find that the electrospinning fibers with opposite polarities will cause a short-circuit when the distance between the two nozzles is $4 \mathrm{~cm}$ [Fig. 9(a)]. $5 \mathrm{~cm}$ is selected as the optimal distance between two nozzles of the bipolarity double-nozzle electrospinning system.

Figure 10 shows the nanofiber motion locus in the opposite-polarity electrospinning process. The two precursor solutions were prepared by mixing polyvinylpyrrolidone (PVP), stannous chloride $\left(\mathrm{SnCl}_{2} \cdot 2 \mathrm{H}_{2} \mathrm{O}\right), \mathrm{N}, \mathrm{N}$-dimethylformamide (DMF), and ethanol, and another precursor solution was prepared by mixing PVP, indium nitrate $\left[\operatorname{In}\left(\mathrm{NO}_{3}\right)_{3} \cdot 4.5 \mathrm{H}_{2} \mathrm{O}\right]$, DMF, and ethanol. The electrostatic voltage $V_{0}$ was $20 \mathrm{kV}$ and the vertical distance $h$ between the nozzles and

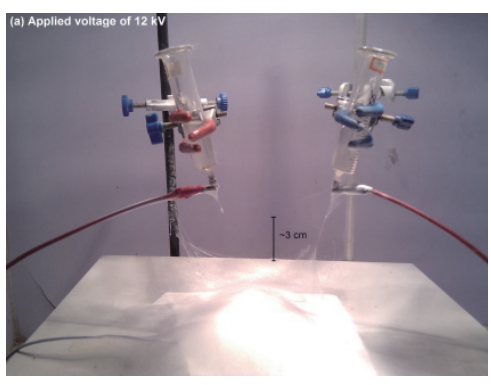

(a)

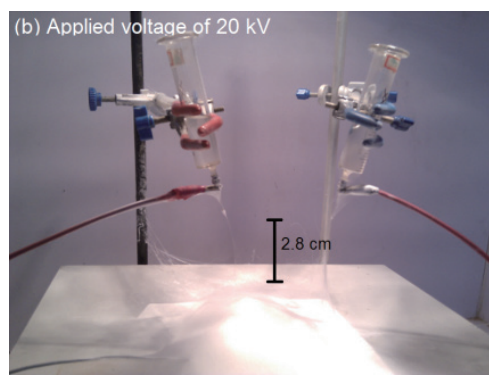

(b)

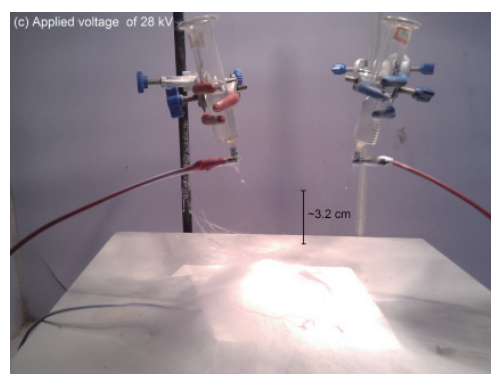

(c)

Fig. 7. (Color online) Electrospinning hetero-nanofiber trajectory at different applied voltages in the bipolarity double-nozzle electrospinning system. Applied voltages of (a) $12 \mathrm{kV}$, (b) $20 \mathrm{kV}$, and (c) $28 \mathrm{kV}$. 


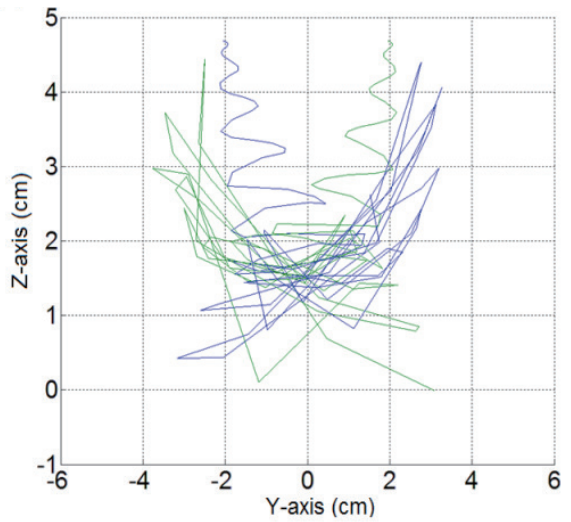

(a)

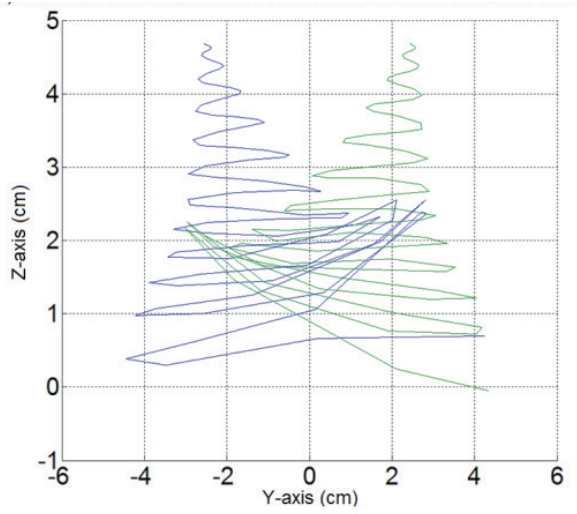

(c)

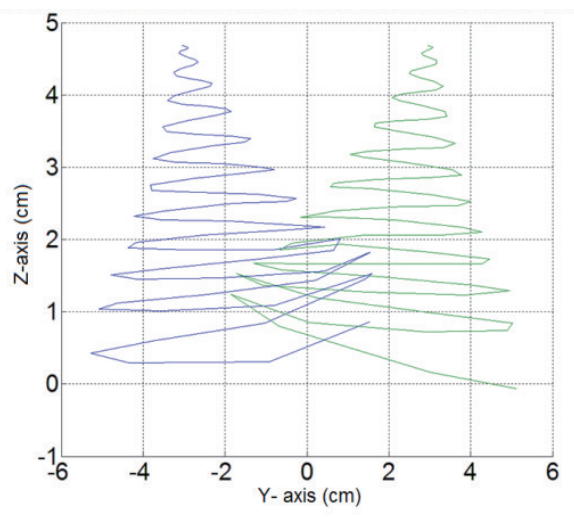

(e)

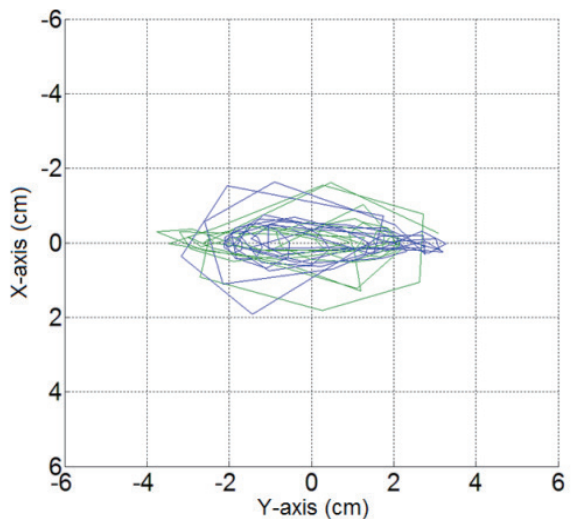

(b)

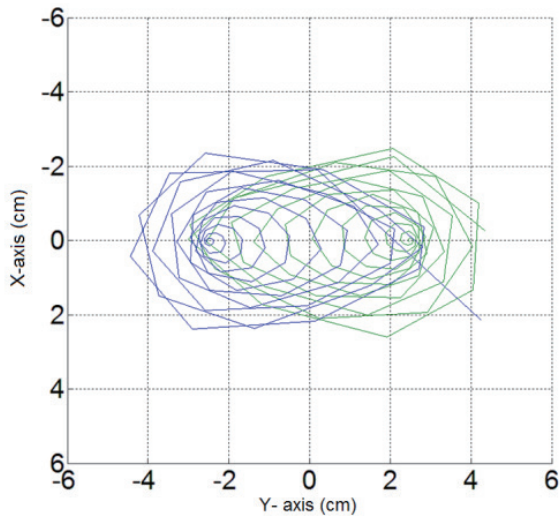

(d)

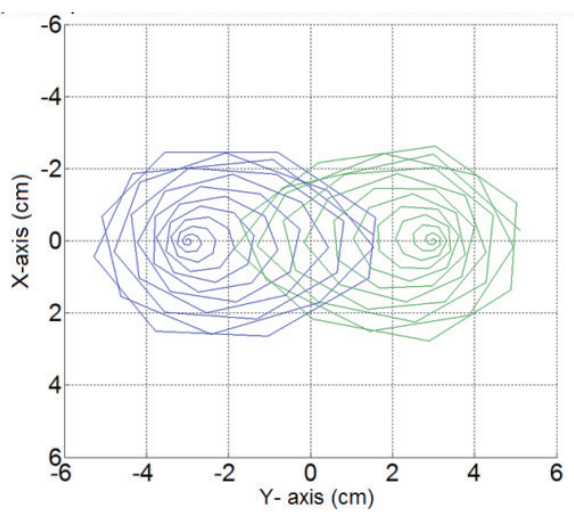

(f)

Fig. 8. (Color online) Simulated motion loci of the jet stream at different electrospinning distances between the two nozzles of the bipolarity double-nozzle electrospinning system. (a) Main view at the distance of $4 \mathrm{~cm}$. (b) Top view at the distance of $4 \mathrm{~cm}$. (c) Main view at the distance of $5 \mathrm{~cm}$. (d) Top view at the distance of $5 \mathrm{~cm}$. (e) Main view at the distance of $6 \mathrm{~cm}$. (f) Top view at the distance of $6 \mathrm{~cm}$.

the collector was $6 \mathrm{~cm}$, and the distance $h_{1}$ between the two nozzles was $5 \mathrm{~cm}$. We can see from Fig. 10 that the electrospinning nanofibers ejected from the two nozzles with opposite polarities moved in opposite directions. After they became attracted to each other and became interwoven, the neutral composite hetero-nanofibers descended to the collection plate because 


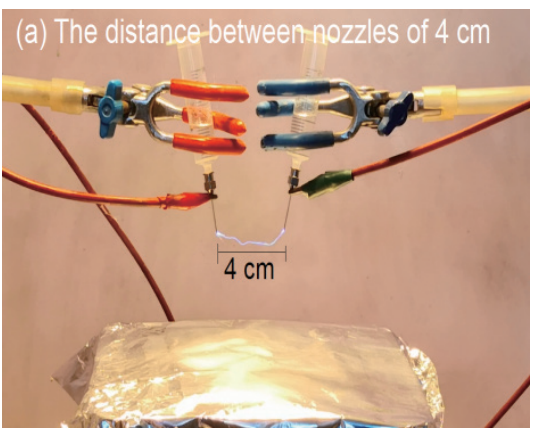

(a)

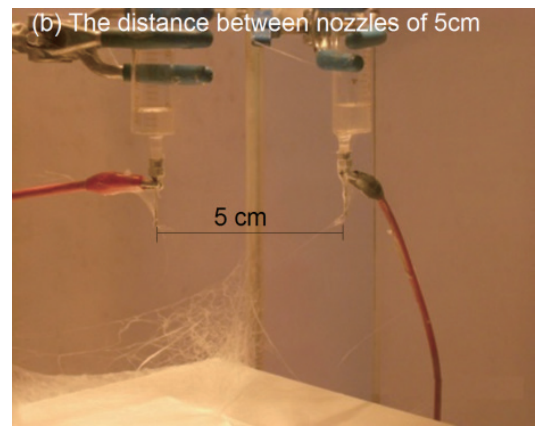

(b)

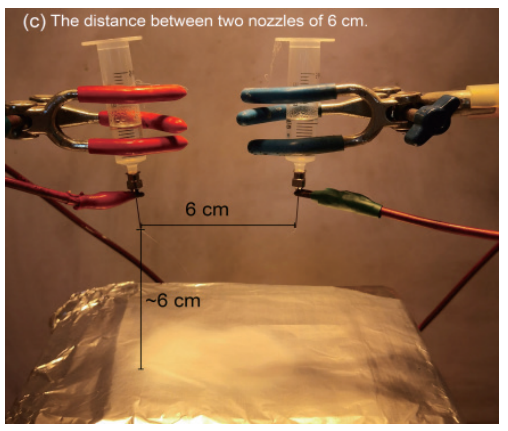

(c)

Fig. 9. (Color online) Electrospinning hetero-nanofiber trajectory at different distances between two nozzles of the bipolarity double-nozzle electrospinning system. (a) Distance between nozzles of $4 \mathrm{~cm}$. (b) Distance between nozzles of $5 \mathrm{~cm}$. (c) Distance between nozzles of $6 \mathrm{~cm}$.

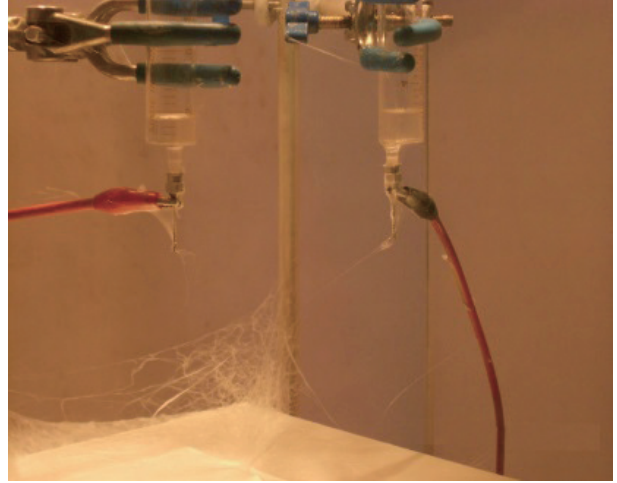

Fig. 10. (Color online) Electrospinning heteronanofiber motion locus using bipolarity double-nozzle electrospinning device.

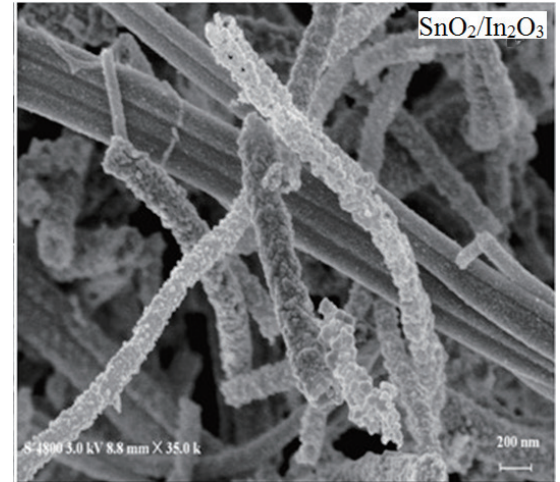

Fig. 11. SEM image of $\mathrm{SnO}_{2} / \mathrm{In}_{2} \mathrm{O}_{3}$ hetero-nanofibers as-synthesized using the bipolarity double-nozzle electrospinning device.

of gravity. The $\mathrm{SnO}_{2} / \mathrm{In}_{2} \mathrm{O}_{3}$ hetero-nanofibers were formed after high-temperature annealing of the previously synthesized organic composite nanofibers. The motion locus of the composite hetero-nanofibers synthesized using a bipolarity double-nozzle electrospinning device is consistent with the simulated motion locus. Figure 11 shows the SEM image of $\mathrm{SnO}_{2} / \mathrm{In}_{2} \mathrm{O}_{3}$ hetero-nanofibers synthesized using the bipolarity double-nozzle electrospinning device. We can see from Fig. 11 that two kinds of nanofibers, $\mathrm{SnO}_{2}$ and $\mathrm{In}_{2} \mathrm{O}_{3}$, exist simultaneously in the $\mathrm{SnO}_{2} / \mathrm{In}_{2} \mathrm{O}_{3}$ composite, presenting a hetero-nanofiber composite system. These two different nanofibers are interlaced to form a net structure.

\section{Summary}

A bipolarity double-nozzle electrospinning device was designed to synthesize composite hetero-nanofibers. The motion locus of the electrospinning nanofibers was simulated to optimize the electrospinning parameters using the MATLAB software. The electrospun solutions were simulated as being multiply charged with discrete particles. Then, force analysis 
was carried out for the charged particles, and a molecular dynamics model was built. The motion locus of the positively charged particles was calculated on the basis of the Runge-Kutta algorithm. The charged particles from the two nozzles with opposite polarities experienced attractive force from each other. Under the combination of electric field forces, Lorentz force, gravity force, surface tension, and viscosity force of solution, two kinds of electrospinning nanofibers were ejected from the nozzles, moved in opposite directions owing to a strong electrical field force and became interwoven, and then descended to the collection plate because of gravity, resulting in the net-structured hetero-nanofibers. Compared with the electrospinning phenomenon under the same experimental conditions, the motion locus of the composite heteronanofibers synthesized using the bipolarity double-nozzle electrospinning system is consistent with the simulated motion locus. The synthesis method based on bipolarity double-nozzle electrospinning was proved to be feasible for synthesizing composite hetero-nanofibers by simulation and experiments. Furthermore, the optimum applied voltages and electrospinning distances were obtained by simulation. More research in simulation and modelling are needed to guide future experiments.

\section{Acknowledgments}

This research was supported in part by the National Natural Science Foundation of China under Grant Numbers 61501081, 61574025, and 61474012, and the Natural Science Foundation of Liaoning Province under Grant Number 2015020096. This research was supported by the Opening Project of Key Laboratory of Microelectronic Devices \& Integrated Technology, Institute of Microelectronics, Chinese Academy of Sciences.

\section{References}

1 Z.-M. Huang, Y. Z. Zhang, M. Kotaki, and S. Ramakrishna: Compos. Sci. Technol. 63 (2003) 2223.

2 N. Bhardwaj and S. C. Kundu: Biotechnol. Adv. 28 (2010) 325.

3 X. Kou, N. Xie, F. Chen, T. Wang, L. Guo, C. Wang, Q. Wang, J. Ma, Y. Sun, H. Zhang, and G. Lu: Sens. Actuators, B 256 (2018) 861.

4 S. K. Lim, S.-H. Hwang, D. Chang, and S. Kim: Sens. Actuators, B 149 (2010) 28.

5 L. Giancaterini, S. M. Emamjomeh, A. De Marcellis, E. Palange, A. Resmini, U. Anselmi-Tamburini, and C. Cantalini: Sens. Actuators, B 229 (2016) 387.

6 J.-A. Park, J. Moon, S.-J. Lee, S.-C. Lim, and T. Zyung: Curr. Appl. Phys. 9 (2009) S210.

7 H. Guan, C. Shao, S. Wen, B. Chen, J. Gong, and X. Yang: Mater. Chem. Phys. 82 (2003) 1002.

8 Y. Wei, Y. Song, X. Deng, B. Han, X. Zhang, Y. Shen, and Y. Lin: J. Mater. Sci. Technol. 30 (2014) 743.

9 Y. He, T. Zhang, W. Zheng, R. Wang, X. Liu, Y. Xia, and J. Zhao: Sens. Actuators, B 146 (2010) 98.

10 F. Ren, L. Gao, Y. Yuan, Y. Zhang, A. Alqrni, O. M. Al-Dossary, and J. Xu: Sens. Actuators, B 223 (2016) 914.

11 H. Du, J. Wang, Y. Sun, P. Yao, X. Li, and N. Yu: Sens. Actuators, B 206 (2015) 753.

12 A. F. Lotus, R. K. Feaver, L. A. Britton, E. T. Bender, D. A. Perhay, N. Stojilovic, R. D. Ramsier, and G. G. Chase: Mater. Sci. Eng., B 167 (2010) 55.

13 H. Zhao, H. Yin, X.-X. Yu, W. Zhang, C. Li, and M.-Q. Zhu: J. Alloys Compd. 735 (2018) 319.

14 P. Lorwongtragool, E. Sowade, N. Watthanawisuth, R. Baumann, and T. Kerdcharoen: Sensors 14 (2014) 19700.

15 K. Sujith, A. M. Asha, P. Anjali, N. Sivakumar, K. R. V. Subramanian, S. V. Nair, and A. Balakrishnan: Mater. Lett. 67 (2012) 376.

16 L. Xu, B. Dong, Y. Wang, X. Bai, Q. Liu, and H. Song: Sens. Actuators, B 147 (2010) 531.

17 Y. Zhang, J. Yang, Q. Li, and X. Cao: J. Cryst. Growth 308 (2007) 180. 
18 P. Wen, M.-H. Zong, R. J. Linhardt, K. Feng, and H. Wu: Trends Food Sci. Technol. 70 (2017) 56.

19 J. W. Huh, H.-J. Jeon, and C. W. Ahn: Curr. Appl. Phys. 17 (2017) 1401.

20 F.-L. He, D.-W. Li, J. He, Y.-Y. Liu, F. Ahmad, Y.-L. Liu, X. Deng, Y.-J. Ye, and D.-C. Yin: Mater. Sci. Eng., C 86 (2018) 18.

21 Z. Chen, X. Cheng, N. Long, H. Zhu, H. Yu, W. Ye, R. Zheng, M. Shui, and J. Shu: Ceram. Int. 44 (2018) 4080.

22 G. Taylor: Proc. R. Soc. London, Ser. A 280 (1964) 383.

23 A. M. Lakdawala, A. Sharma, and R. Thaokar: Chem. Eng. Sci. 148 (2016) 238.

24 M. Joulaian, A. Pishevar, S. Khajepor, F. Schmid, and Y. Afshar: Comput. Phys. Commun. 183 (2012) 2405.

25 X. Song, W. Liu, J. Wang, S. Xu, B. Liu, Q. Cai, and Y. Ma: Mater. Lett. 212 (2018) 20.

26 J. Lasprilla-Botero, M. Álvarez-Láinez, and J. M. Lagaron: Mater. Today Commun. 14 (2018) 1.

27 M. Rabionet, T. Puig, and J. Ciurana: Procedia CIRP 65 (2017) 267.

28 N. Ismail, F. J. Maksoud, N. Ghaddar, K. Ghali, and A. Tehrani-Bagha: J. Aerosol Sci. 113 (2017) 227.

29 D. G. Venkateshan, M. A. Tahir, H. Vahedi Tafreshi, and B. Pourdeyhimi: Mater. Des. 96 (2016) 27.

30 D. N. Riahi: Int. Non Linear Mech. 92 (2017) 1.

31 D. A. Saville: J. Fluid Mech. 48 (2006) 815.

32 M. Yousefzadeh: 12 - Modeling and Simulation of the Electrospinnng Process: In Electrospun Nanofibers (Woodhead Publishing, 2017) p. 277.

33 G. Campatelli, L. Sapuppo, and A. Scippa: Simul. Modell. Pract. Theory 52 (2015) 135.

34 M. Su, J. Wang, H. Du, P. Yao, Y. Zheng, and X. Li: Sens. Actuators, B 161 (2012) 1038.

35 H. Du, J. Wang, M. Su, P. Yao, Y. Zheng, and N. Yu: Sens. Actuators, B 166-167 (2012) 746.

36 M. Lauricella, G. Pontrelli, D. Pisignano, and S. Succi: J. Comput. Sci. 17, Part 2 (2016) 325.

37 Y. Yang, Z. Jia, Q. Li, L. Hou, H. Gao, L. Wang, and Z. Guan: Int. Conf. Properties and Applications of Dielectric Materials (2006) 940.

38 I. Nezbeda, J. Jirsák, and F. Moučka: 11 - Molecular Modeling and Simulations: In Electrospun Nanofibers (Woodhead Publishing, 2017) p. 255.

39 R. P. A. Hartman, D. J. Brunner, D. M. A. Camelot, J. C. M. Marijnissen, and B. Scarlett: J. Aerosol Sci. 31 (2000) 65.

40 D. H. Reneker, A. L. Yarin, H. Fong, and S. Koombhongse: J. Appl. Phys. 87 (2000) 4531.

41 Z. Liu, J. Wang, D. U. Haiying, H. Wang, L. I. Xiaogan, and X. Wang: Chin. J. Mater. Res. 32 (2018) 127. 